\title{
Analysis of membraneless formic acid microfuel cell using a planar microchannel
}

\author{
Falin Chen $^{\mathrm{a}}$, Min-Hsing Chang ${ }^{\mathrm{b}, *}, \mathrm{Mu}-\mathrm{Kun}$ Lin $^{\mathrm{a}}$ \\ ${ }^{a}$ Institute of Applied Mechanics, National Taiwan University, Taipei 10764, Taiwan, Republic of China \\ ${ }^{\mathrm{b}}$ Department of Mechanical Engineering, Tatung University, 40, Chun-Shan North Rd., Taipei 104, Taiwan, Republic of China
}

Received 20 June 2006; received in revised form 26 August 2006; accepted 4 September 2006

Available online 2 October 2006

\begin{abstract}
A novel design of membraneless microfuel cell employing a planar microchannel has been investigated theoretically in this study. The fuel and oxidant are, respectively, formic acid and oxygen and both dissolved in dilute sulfuric acid solutions. Both liquid streams enter the planar microchannel and flow in parallel without the need of a membrane to separate them. A theoretical model is developed to simulate the species transport in both anode and cathode streams and the cell performance is analyzed accordingly by examining the effects of flow rate, concentration, and the geometric size of the system. The results show that the cell performance is mainly restricted by the high transport resistance in the cathode stream. It is found that the transport of oxygen to the cathode electrode can be improved significantly by using a higher flow rate or oxygen concentration, or a thicker cathode catalyst layer. However, the effectiveness of the flow rate and thickness of catalyst layer diminishes gradually which indicates that there exist optimal conditions of these parameters. The influences of thickness and length of the microchannel on cell performance are also examined in detail.
\end{abstract}

(C) 2006 Elsevier Ltd. All rights reserved.

Keywords: Membraneless microfuel cell; Planar microchannel; Formic acid

\section{Introduction}

Small fuel cells have received much attention recently due to their widely promising applications in portable power sources [1-13]. One of the major technique problems about the fulfillment of practical application is to effectively reduce the volume occupied by the whole system. Traditional proton exchange membrane fuel cell (PEMFC) or direct methanol fuel cell (DMFC) is difficult to be miniaturized because of its complicated system structures. Therefore, it is vital to search for a possible alternative design of small fuel cell to be used in miniature portable power systems such as cell phones, laptop computers, and MEMs related devices. Recently, a novel design of membraneless microfuel cell has been proposed and investigated by many researchers [1-9]. Both liquid fuel and oxidant streams enter and flow in parallel through the microchannel between two

\footnotetext{
* Corresponding author. Tel.: +8862 25925252×3410; fax: +8862 25997142 .

E-mail addresses: mhchang@ttu.edu.tw, mhchang@mail.ytit.edu.tw (M.-H. Chang).
}

electrodes. Under small Reynolds number conditions, the occurrence of laminar flow naturally separates both streams without turbulent mixing and eliminates the need of a membrane to partition them. Such a novel design obviously possesses many merits in comparison with conventional PEMFC [2]: the ohmic losses and fouling problems due to the membrane are removed, the system structure and fabrication are simpler, the water management is much easier, and most significantly the size of fuel cell can be miniaturized effectively. Moreover, the liquid fuels have higher energy densities over gaseous fuels, which is quite an important property in the applications of portable power sources [3].

Ferrigno et al. [1] first proposed a membraneless redox fuel cell using a Y-shaped millimeter-scale channel. This design used the same species (vanadium) as oxidant and reductant, and was found to be comparable in its performance to a macro-system. However, its efficiency of fuel utilization was quite poor which was thought to be limited primarily by mass transport to the electrodes. Choban et al. [2] further designed a microfluidic fuel cell utilizing a Y-shaped microchannel. The fuel used in this work is formic acid and the oxidant is oxygen dissolved in sulfuric acid solution. They found that the cell performance is restrained 
profoundly by the transport of reactants through the concentration boundary layer adjacent to the electrodes and the low concentration of oxygen in cathode stream. The fuel utilization was still quite low in this design that is less than $1.0 \%$. Theoretical analyses based on this design have been performed by Bazylak et al. [3] and Chang et al. [4]. The former considered a Tshaped membraneless microfuel cell and focused on how to raise the efficiency of fuel utilization. Their numerical results suggested that an extended tapered-electrode design can improve the fuel utilization greatly. Chang et al. [4] developed a theoretical model for a Y-shaped configuration as the design of Choban et al. [2] and analyzed the cell performance with a systematic study by considering many important system parameters. Their results are in agreement with the experimental observations [2] and predict that the cell performance can be improved by using a high aspect ratio of cross-sectional geometry. Choban et al. [5] further investigated the characterization of performance limiting factors including the kinetics and mass transfer limitations in their design [2]. They continued to study the effects of media flexibility on the reaction kinetics [6] by working with acidic media in anode stream and alkaline media in cathode stream, or vice versa. A new design of air-breathing laminar flow-based microfluidic fuel cell [7] to enhance the replenishment of oxygen in cathode stream was also reported by them.

In contrast to the Y-shaped microchannel used in the works [1-6], a novel design of planar membraneless microchannel fuel cell was presented by Cohen et al. [8,9]. Such a planar design have many advantages over the Y-shaped model: the deposition of electrode materials is more manageable, the contact areas between the electrodes and fuel and oxidant streams are larger, and the stacking of devices is easier to lead to high power system occupying small volume. The formic acid system was used in their experiments [8] and the open circuit potential as well as the kinetic behavior was observed and compared to those of macro-fuel cell systems. They further investigated the case of a dual electrolyte $\mathrm{H}_{2} / \mathrm{O}_{2}$ planar membraneless microfuel cell [9] to achieve a higher open circuit voltage.

Since the planar design of membraneless microfuel cell gives rise to larger contact areas between the electrodes and electrolytes and seems potentially to be easier to constitute a cell stack, it is important to understand the kinetics and transport characterizations of reactants in such a planar microchannel in order to reach the goal of optimal design. In this study, we develop a theoretical model to simulate the planar membraneless microfuel cell system including the effects of flow kinetics, species transport, and electrochemical reactions at the electrodes. A 3-D numerical analysis is performed to explore the influences of critical system parameters such as flow rate, species concentration, and geometric size of microchannel, on cell performance. The results provide a complete overview for the characteristics of this kind of membraneless microfuel cell employing a planar microchannel design.

\section{Theoretical model and formulation}

The side view of the planar membraneless microfuel cell is shown in Fig. 1. Both fuel and oxidant streams enter the pla-

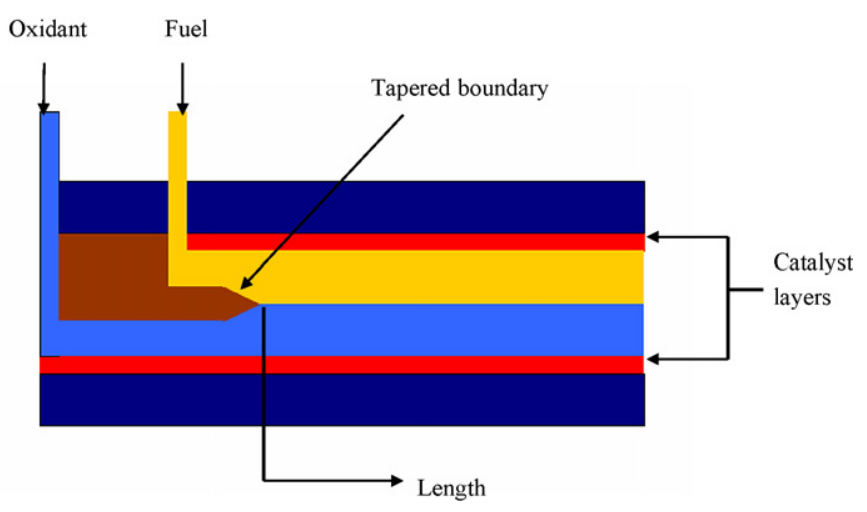

Fig. 1. The side view of the planar membraneless microfuel cell.

nar microchannel at two separate inlets, and then merge after the tapered flow boundary. The system dimensions are listed in Table 1. The fuel and oxidant are, respectively, formic acid and oxygen, and both are in $0.1 \mathrm{M}$ sulfuric acid solutions. A thin porous catalyst layer adheres to the upper anode surface as well as the below cathode surface. Electrochemical reactions are assumed to occur in these catalyst layers only. At the anode, the reaction is

$\mathrm{HCOOH} \rightarrow \mathrm{CO}_{2}+2 \mathrm{H}^{+}+2 \mathrm{e}^{-}$,

where formic acid releases energy and creates protons and electrons. The electrons move to the cathode via an external circuit and the protons diffuse across the cathode interface of both streams into the cathode flow. At the cathode, the oxygen reacts with the protons in the stream and the electrons coming from the anode and results in the reaction

$\mathrm{O}_{2}+4 \mathrm{H}^{+}+4 \mathrm{e}^{-} \rightarrow 2 \mathrm{H}_{2} \mathrm{O}$

Note that the products, carbon dioxide and water, can be removed simply by the liquid streams. Assume that the system is isothermal and both liquid streams are steady incompressible flows. Accordingly, in both fluid layers the transports of mass, momentum, and concentration can be described by the general continuity equation, Navier-Stokes equation, and Fick's law, respectively. While in the catalyst layers, it is assumed that the porous electrodes are isotropic and can be defined by the Darcy equation. The corresponding conductivities are taken to be related to the effective solid-phase conductivity by the Bruggeman-type relation with tortuosity 1.5 [14]. Since the electrochemical reactions occur in these layers, the concentration

Table 1

The dimensions of the system

\begin{tabular}{lr}
\hline Width $(\mathrm{mm})$ & 1.0 \\
Length $(\mathrm{cm})$ & 5.0 \\
Inlet width $(\mathrm{mm})$ & 1.0 \\
Anode stream thickness $(\mu \mathrm{m})$ & 190.0 \\
Cathode stream thickness $(\mu \mathrm{m})$ & 190.0 \\
Catalyst layer thickness $(\mathrm{nm})$ & 100.0 \\
\hline
\end{tabular}


equation can be written as

$u_{j} \frac{\partial C_{i}}{\partial x_{j}}=D_{i} \Delta C_{i}+\frac{e_{i}}{n F} S_{i}$,

where the subscript ' $i$ ' represents the reactant species, $u_{j}$ the velocity component with $j=1-3, C$ the concentration, $D$ the diffusion coefficient, $\Delta$ the Laplacian operator, $e$ the mole number, $n$ the number of electrons transferred in the reaction, $F$ the Faraday constant, and $S$ is the rate of electrochemical reaction in terms of the volumetric rate of current generation with unit $\mathrm{A} \mathrm{m}^{-3}$. The electric potential equation in both liquid streams can be defined by

$\sigma_{i} \Delta \Phi_{i}=S_{i}$

where we assume the electric conductivity $\sigma$ is a constant and $\Phi$ the electric potential. Note that the reaction rate $S_{i}$ is zero in the liquid layers and related to the reactant concentration in the catalyst layers by the Bulter-Volmer equation

$S_{i}=a j_{0}\left(\frac{C_{i}}{C_{i}^{0}}\right)^{\beta_{i}}\left[\exp \left(\frac{\alpha_{\mathrm{a}} n F}{R T} \eta\right)-\exp \left(-\frac{\alpha_{\mathrm{c}} n F}{R T} \eta\right)\right]$,

where $a$ is the density of the catalyzed active area on the electrode, $j_{0}$ the exchange current density at the reference reactant concentration $C_{i}^{0}, C_{i}$ the concentration of species $i, \beta_{i}$ the reaction order, $\alpha_{\mathrm{a}}$ and $\alpha_{\mathrm{c}}$ the charge transfer coefficient, respectively, for the anodic and cathodic reactions, $R$ the universal gas constant, $T$ the absolute temperature, and $\eta$ is the overpotential.

The boundary conditions at the inlets are given by the assigned pressure, velocity, and concentration. The pressure at the exit is given by the atmospheric pressure. At the interface between both liquid streams, the velocity, concentration, and concentration flux are assumed to be continuous. At the liquid-porous interfaces, the normal velocity, concentration, and concentration flux are also continuous across each interface. Once the theoretical mode is solved, the vector of current density $j$ can be determined by

$j_{i}=-\sigma \frac{\partial \Phi}{\partial x_{i}}$

in which the component normal to the electrode surface is the only useful one to contribute to the output of electricity of fuel cell. The commercial CFD package, CFD-RC, was employed to perform the numerical analysis. The system was discretized with structured grids and solved by the upwind scheme based on the finite volume method. The grid tests had been made to insure the numerical accuracy and obtain converged results with economic consumption of CPU time.

\section{Results and discussion}

We first compare the computational results with those of experimental study [8]. Both cases are considered that the oxidant is air-saturated and aerated with oxygen gas as those investigated in Ref. [8]. Hence, we use the oxygen concentration $0.25 \mathrm{mM}$ in an air-saturated solution at room temperature, and
Table 2

The parameters used in the base state

\begin{tabular}{ll}
\hline Anode side: & \\
Inlet flow rate $(\mathrm{ml} / \mathrm{min})$ & 0.5 \\
Fuel concentration $(\mathrm{M})$ & 0.5 \\
Conductivity $(\mathrm{mho} / \mathrm{m})$ & 5 \\
Density $\left(\mathrm{kg} / \mathrm{m}^{3}\right)$ & 1008.6 \\
Diffusivity $\left(\mathrm{m}^{2} / \mathrm{s}\right)$ & $2.546 \times 10^{-9}$ \\
Porosity of catalyst layer & 0.5 \\
Exchange current density $\left(\mathrm{A} / \mathrm{m}^{2}\right)$ & 0.1194 \\
Cathode side: & \\
Inlet flow rate $(\mathrm{ml} / \mathrm{min})$ & 0.5 \\
Oxygen concentration $(\mathrm{mM})$ & 1.25 \\
Conductivity $(\mathrm{mho} / \mathrm{m})$ & 4.78 \\
Density $\left(\mathrm{kg} / \mathrm{m}^{3}\right)$ & 1004.9 \\
Diffusivity $\left(\mathrm{m}^{2} / \mathrm{s}\right)$ & $2.1 \times 10^{-9}$ \\
Porosity of catalyst layer & 0.5 \\
Exchange current density $\left(\mathrm{A} / \mathrm{m}^{2}\right)$ & $3.1 \times 10^{-7}$ \\
\hline
\end{tabular}

assume that the oxygen concentration in the oxygen-aerated oxidant stream is equal to $1.25 \mathrm{mM}$ that is the value for a oxygen saturated solution, by using the parameters' list in Table 2 and under the same flow rate condition, it is found that the numerical results are in good agreement with those of experimental findings [8] as shown in Fig. 2. Accordingly, the present results could provide not only qualitatively but also quantitatively theoretical predictions. In the following paragraphs, we will take the condition list in Table 2 with oxygen concentration $1.25 \mathrm{mM}$ as the base state. The effects of each parameter including flow rate, concentration, catalyst layer, and channel size, on the transport characteristics of reactants in both anode and cathode streams as well as the cell performance will be described in detail based on this condition.

\subsection{Effects of volumetric flow rate}

The volumetric flow rate, $Q$, plays an important role in the performance of membraneless laminar flow-based microfuel cell, where $Q$ is the combination of equal flow rates of the fuel and oxidant streams in the planar microchannel. If $Q$ is too low, both fuel and oxidant streams will have more time to be in diffusional contact and thus cause a higher degree of mixing. Fig. 3(a-f) shows the concentration profiles of formic acid and oxygen at

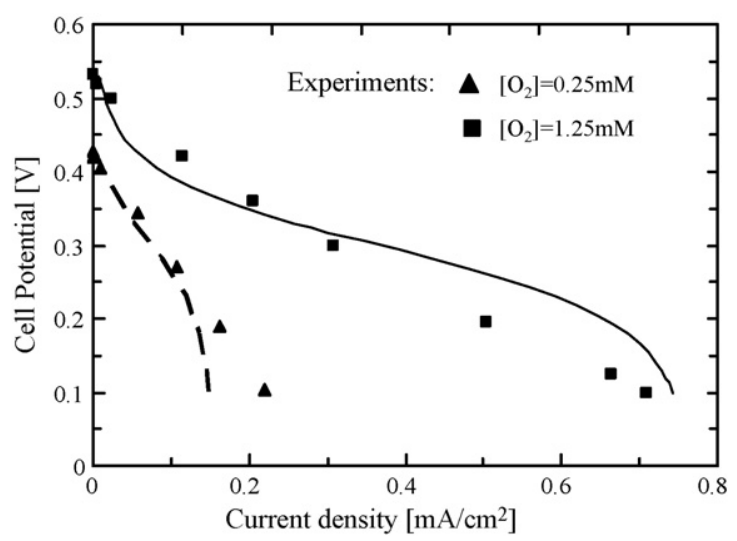

Fig. 2. Comparisons of cell performance with the experimental results of Cohen et al. [8]. 


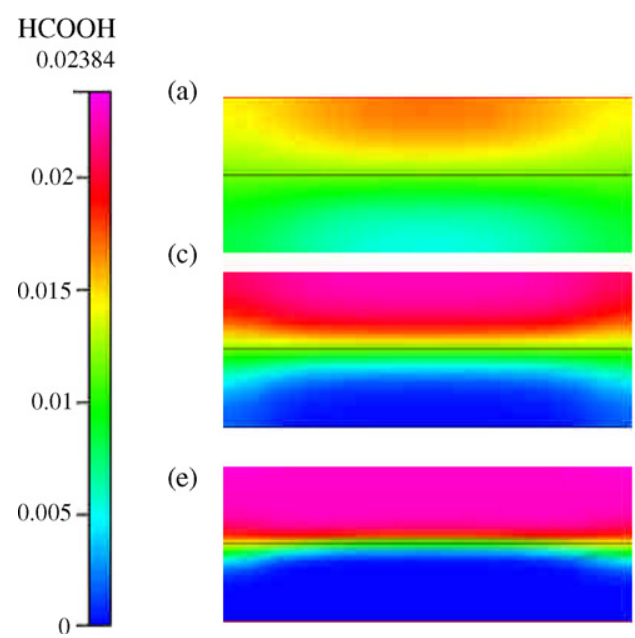

(b)

(b)

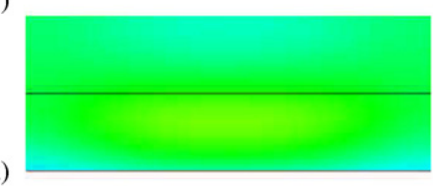

(d)

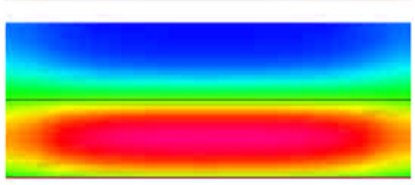

(f)

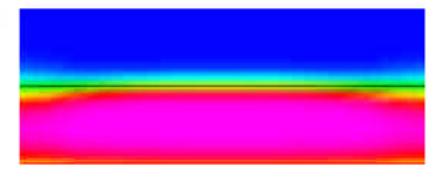

02

3.767E-005

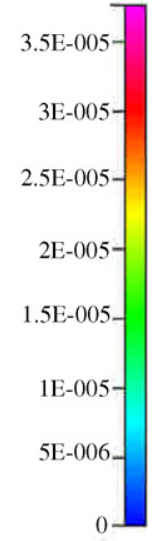

Fig. 3. The concentration contours of formic acid and oxygen at the exit of microchannel for three assigned flow rate $Q$ at cell potential $0.3 \mathrm{~V}$ : (a) formic acid with $Q=0.2 \mathrm{ml} / \mathrm{min}$, (b) oxygen with $Q=0.2 \mathrm{ml} / \mathrm{min}$, (c) formic acid with $Q=1.0 \mathrm{ml} / \mathrm{min}$, (d) oxygen with $Q=1.0 \mathrm{ml} / \mathrm{min}$, (e) formic acid with $Q=8.0 \mathrm{ml} / \mathrm{min}$, and (f) oxygen with $Q=8.0 \mathrm{ml} / \mathrm{min}$.

the microchannel exit for three typical flow rates. As shown in Fig. 3( $\mathrm{a}$ and $\mathrm{b}$ ) for the case $Q=0.2 \mathrm{ml} / \mathrm{min}$, one can see that there is no distinct interface between both streams and both solutes, formic acid and oxygen, diffuse each other making the diffusion zone almost occupy the entire channel cross-section. As a result, the diffusive transport becomes dominant and produces a severe fuel crossover phenomenon. It results in a strong mixed potential and lower the cell performance significantly. As the flow rate $Q$ increases to $1.0 \mathrm{ml} / \mathrm{min}$ as shown in Fig. 3(c and d), a distinct diffusion layer appears between the upper anodic stream and the lower cathodic stream. The thickness of this layer is obviously larger in the regions adjacent to the lateral walls, which is caused by the lower velocities in the convective boundary layers where the solutes could have more time to diffuse and mix. These results are consistent with the experimental findings [13] in a similar Y-shaped microchannel. In Fig. 3(d) we also observed that there exists an apparent depletion zone in the bottom of cathode stream near the electrode surface. This zone is the concentration boundary layer of oxygen caused by the consumption of oxidant in the cathode electrode. The appearance of this zone indicates that the transport of oxygen is too low to provide sufficient oxygen flux toward the cathode electrode. Whereas as seen in Fig. 3(c), the depletion zone on the surface of anode electrode is negligible because of the high concentration of formic acid solution over that of oxygen solution in cathode stream. These results reveal that the cell performance in such a planar design is still dominant by the transport of oxygen to cathode electrode which is the same as the characteristic of Y-shaped microchannel design. As the flow rate increases to $8.0 \mathrm{ml} / \mathrm{min}$ as shown in Fig. 3(e and f), the diffusion layer between both streams as well as the depletion zone on the cathode electrode surface becomes thinner. As a result, the mass flux of oxygen to cathode is enhanced and thus the cell performance can be improved significantly. The corresponding $I-V$ and power density curves are demonstrated in Fig. 4(a and b), respectively. Obviously, the cell performance and power density rise gradually with the flow rate. However, the limiting current density of each curve in Fig. 4(a) is not proportional to the flow rate as well as the maximum power density in Fig. 4(b). For example, in Fig. 4(a), the limiting current density changes from 0.73 to $1.18 \mathrm{~mA} / \mathrm{cm}^{2}$ only as the flow rate increase eight times from 1.0 to $8.0 \mathrm{ml} / \mathrm{min}$. These results indicate that although a stronger convective flow may enhance the transport of reactants to the electrodes, this effect diminishes gradually and the concentra-
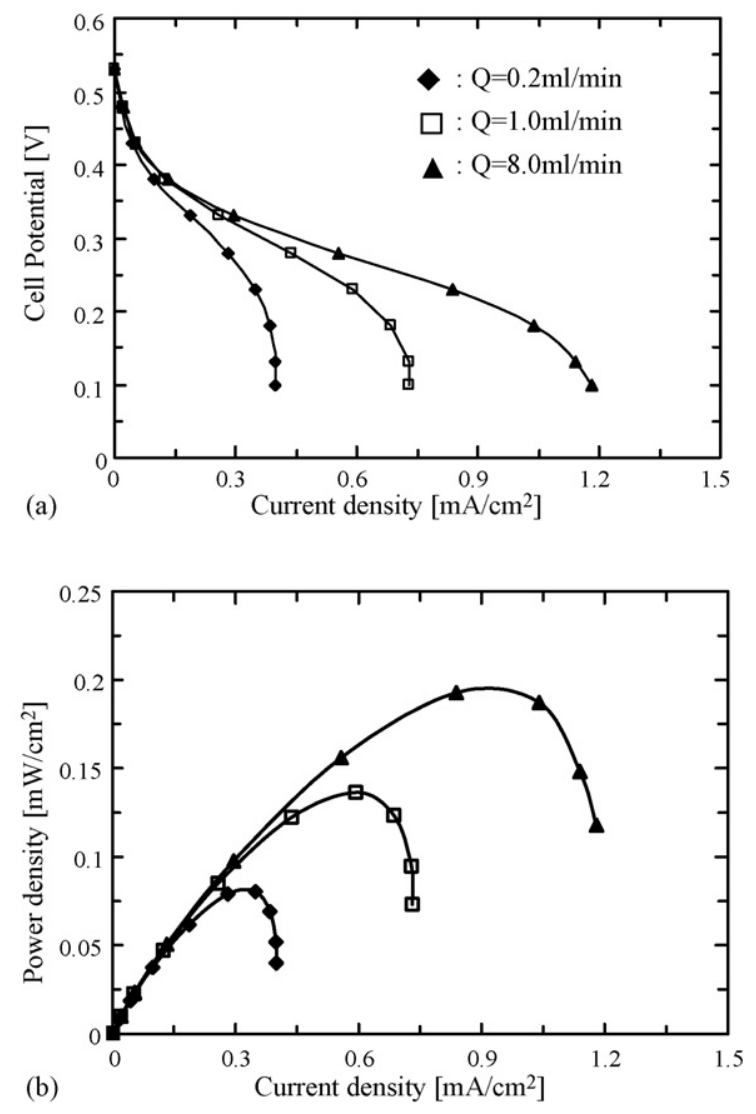

Fig. 4. (a) The $I-V$ curves for three assigned volumetric flow rate $Q$; (b) the corresponding power density curves. 
tion loss due to the insufficient transport of oxygen to the cathode electrode surface occurs eventually.

\subsection{Effects of concentrations of reactants}

To examine the effects of concentration on cell performance, we first consider the concentration of formic acid in anode stream. It is expected that its influence should be small since the cell performance is primarily cathode-limited. The results confirm this expectation and find that if the fuel used is $1.0 \mathrm{M}$ formic acid solution, the corresponding $I-V$ curve will be quite close to that of base condition with $0.5 \mathrm{M}$ formic acid solution as shown in Fig. 4(a) and the limiting current density will increase $0.034 \mathrm{~mA} / \mathrm{cm}^{2}$ only. However, the cell performance is very sensitive to the variation of oxygen concentration in cathode stream. Fig. 5 illustrates the $I-V$ curves for these assigned values of oxygen concentration. Obviously, the cell performance can be improved significantly by using a higher oxygen concentration solution. Note that the limiting current density is approximately proportional to the oxygen concentration. Thus, it could be the most efficient way to promote the cell performance by aerating more oxygen into the cathode stream. The oxidant consumption rate is also an important property for this planar design of membraneless microfuel cell. The oxidant consumption rate, $\lambda$, is defined as

$\lambda=\frac{\dot{m}_{\mathrm{i}}-\dot{m}_{\mathrm{e}}}{\dot{m}_{\mathrm{i}}} \times 100 \%$,

where $\dot{m}_{\mathrm{i}}$ and $\dot{m}_{\mathrm{e}}$ are the mass flow rate of oxygen at the inlet and outlet of the microchannel, respectively. The variations of $\lambda$ with volumetric flow rate $Q$ are shown in Fig. 6 for those three typical oxygen concentrations. It is found that the consumption rate is quite high at low flow rate condition since the oxygen has more time to be transported and react with the electrode. However, the diffusion mixing is severe under such a condition and makes a great amount of fuel crossover. Hence, the cell performance is quite low. As the flow rate increases, the value of $\lambda$ decreases quickly first and then gradually approaches a constant about $4.3 \%$. This indicates that, as the magnitude of convective flow is large enough, the transport of oxygen to the electrode surface reaches a maximum and the consumption rate is dominated by the rate of electrochemical reaction in the catalyst layer.

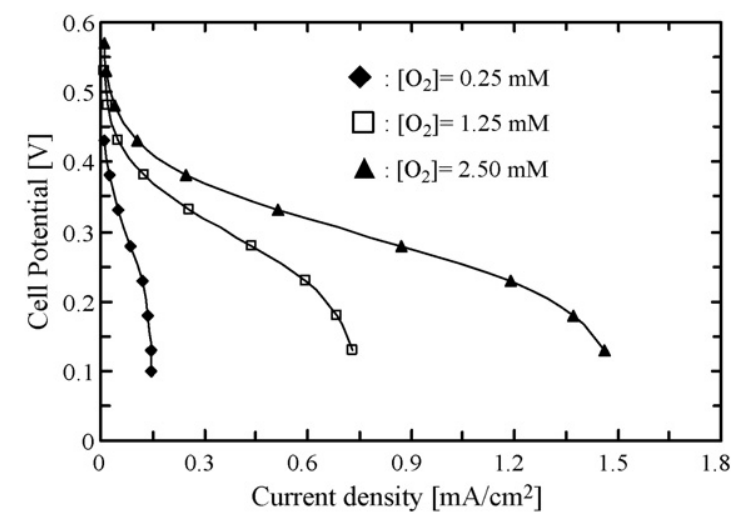

Fig. 5. The $I-V$ curves for three assigned concentrations of oxygen.

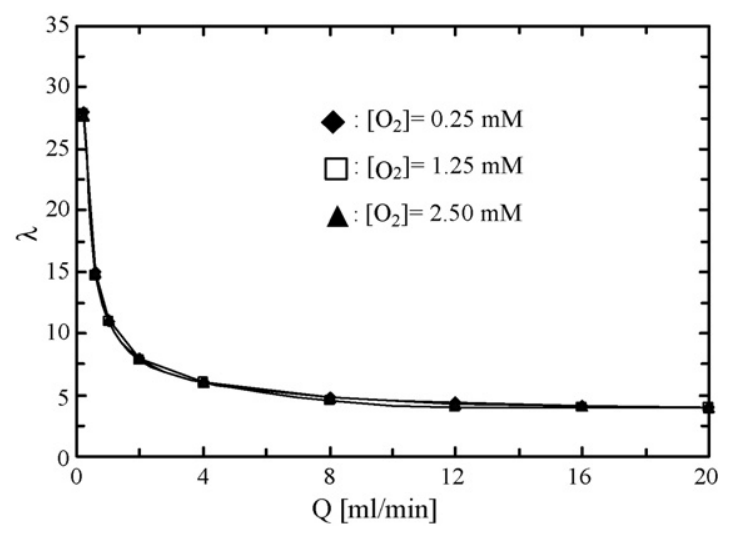

Fig. 6. The variations of oxidant consumption rate $\lambda$ with volumetric flow rate $Q$ for three assigned concentrations of oxygen.

Furthermore, the three curves almost overlap which reveals that the consumption rate is independent of the oxygen concentration although the mass flux increases for a higher oxygen concentration.

\subsection{Effects of thicknesses and porosities of catalyst layers}

Because the cell performance is obviously cathode-limited, we first pay attention to the effects of cathode catalyst layer. Assuming that this layer was made by the deposition of platinum particles uniformly on a thin porous substrate, the $I-V$ curves for three typical thicknesses $h_{\mathrm{c}}$ of this layer are demonstrated in Fig. 7. One can see that the cell performance rises significantly when the thickness $h_{\mathrm{c}}$ increases from 100 to $500 \mathrm{~nm}$. But if $h_{\mathrm{c}}$ increases further from 500 to $900 \mathrm{~nm}$, the improvement is relatively limited which indicates that there exists a optimal value of $h_{\mathrm{c}}$ about $500 \mathrm{~nm}$ in the present base case. If one uses a higher thickness than this value, it seems to waste material and produces little contribution on cell performance only.

Fig. 8 shows the variations of limiting current density $I_{1}$ with volumetric flow rate $Q$ for these three typical values of $h_{\mathrm{c}}$. Each curve rises rapidly at first due to the reduction of fuel crossover and the depletion zone on the electrode surface as indicated in Fig. 3, and then tends to be a constant eventually. It is noted that even at high flow rate states, the effects of increasing $h_{\mathrm{c}}$ on cell performance still decay gradually. Accordingly, the optimal

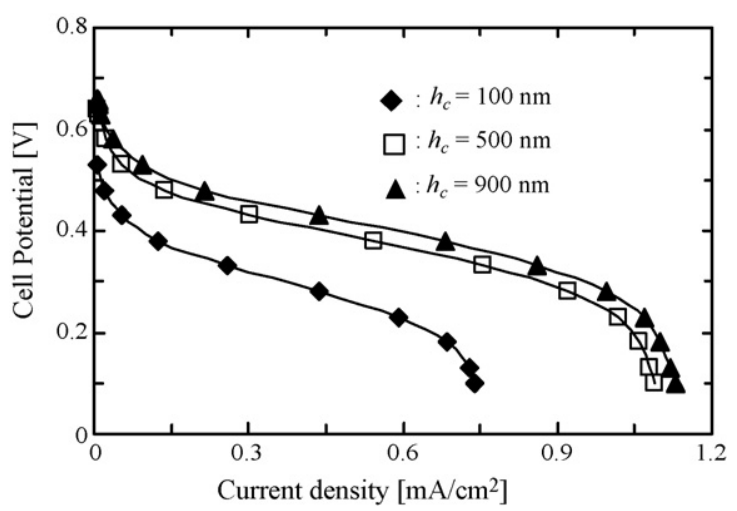

Fig. 7. The $I-V$ curves for three assigned thicknesses of cathode catalyst layer. 


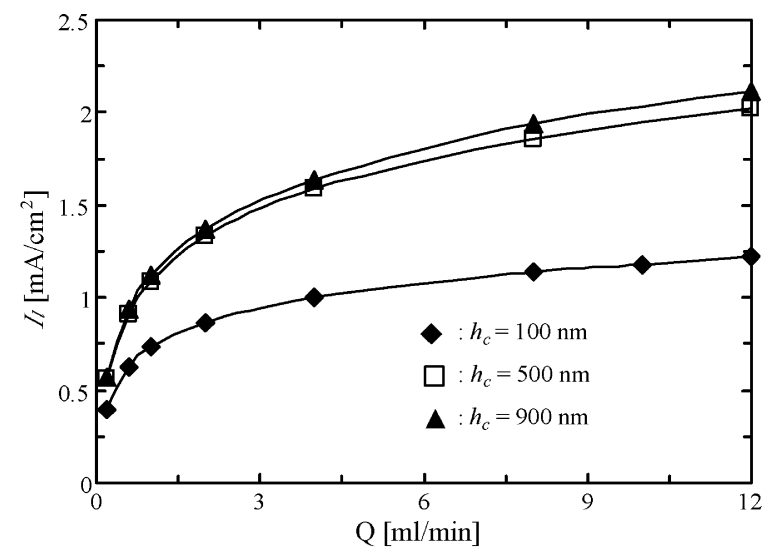

Fig. 8. The variations of limiting current density $I_{1}$ with volumetric flow rate $Q$ for three assigned values of thickness of cathode catalyst layer $h_{\mathrm{c}}$.

value of $h_{\mathrm{c}}$ is independent of the flow rate $Q$. However, if we change the other properties of cathode stream such as the concentration of oxygen or sulfuric acid, it would be important to determine the best condition of cathode catalyst layer.

In order to understand the influences of $h_{\mathrm{c}}$ on the transport of oxygen to the cathode electrode, the concentration profiles of oxygen at the outlet of microchannel with these three values of $h_{\mathrm{c}}$ are illustrated in Fig. 9(a-f) for two typical flow rate $Q=1.0$ and $8.0 \mathrm{ml} / \mathrm{min}$. As shown in Fig. 9(a-c) for the case $Q=1.0 \mathrm{ml} / \mathrm{min}$, it is observed that the concentration boundary layer adjacent to the bottom electrode is quite apparent in Fig. 9(a) at $h_{\mathrm{c}}=100 \mathrm{~nm}$ and it becomes larger in Fig. 9(b) for $h_{\mathrm{c}}=500 \mathrm{~nm}$. While in Fig. 9 (c) for $h_{\mathrm{c}}=900 \mathrm{~nm}$, the thickness of the concentration boundary layer appears almost the same as that of Fig. 9(b). Therefore, it is evident that the consumption rate of oxygen of the case Fig. 9(a) is lower than those of Fig. 9(b and c). We also observe that the diffusive mixing is quite strong especially in the regions near the lateral walls in all three cases. In the case $Q=8.0 \mathrm{ml} / \mathrm{min}$ as shown in Fig. $9(\mathrm{~d}-\mathrm{f})$, the thicknesses of the concentration boundary layers on the surfaces of bottom

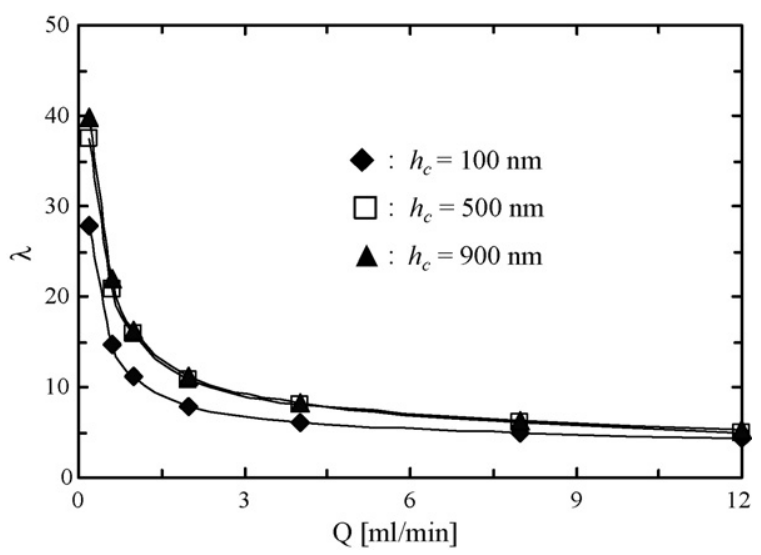

Fig. 10. The variations of oxidant consumption rate $\lambda$ with volumetric flow rate $Q$ for three assigned values of $h_{\mathrm{c}}$.

electrodes becomes relatively small but still appear to have the same variational behaviors with $h_{\mathrm{c}}$. Evidently, the consumption rate of oxygen is higher at lower flow rate but the severe losses due to fuel crossover lower the cell performance. In contrast, at higher flow rate, the consumption rate of oxygen is lower but a better performance can be achieved due to the great reduction of fuel crossover. The corresponding variations of oxygen consumption rate $\lambda$ with flow rate $Q$ are demonstrated in Fig. 10 for these typical values of $h_{\mathrm{c}}$. It is noticed that at the given flow rate $Q=8.0 \mathrm{ml} / \mathrm{min}$, the value of $\lambda$ increases from 4.9 to $6.2 \%$ only as the thickness $h_{\mathrm{c}}$ changes from 100 to $500 \mathrm{~nm}$, and then appears to be a constant as $h_{\mathrm{c}}$ increases further to $900 \mathrm{~nm}$. This result confirms that the optimal value of $h_{\mathrm{c}}$ is about $500 \mathrm{~nm}$. Besides, it should be vital trying to find a more efficient way to improve the utilization rate of oxygen under a constant flow rate. Similarly, the influences of anode catalyst layer also have been investigated in detail. It is found that the $I-V$ curve does not get affected by the thickness of anode catalyst at all. This result also verifies that the cell performance is mainly dominated by the cathode side. (a)

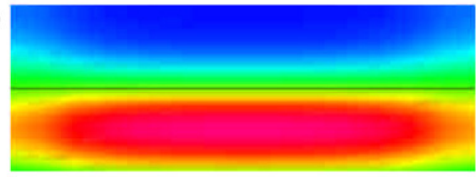

(b)

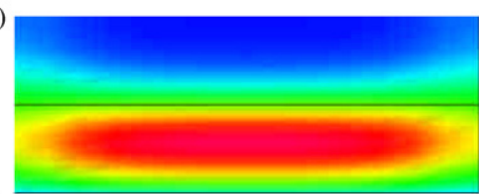

(c)

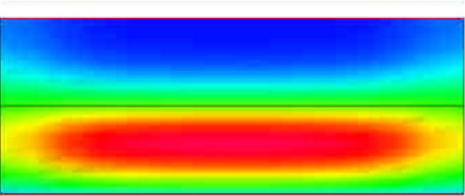

(d)

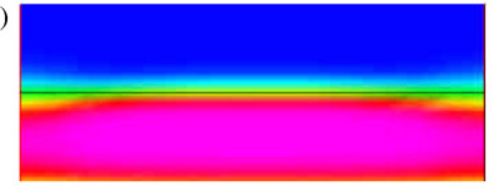

(e)

(f)
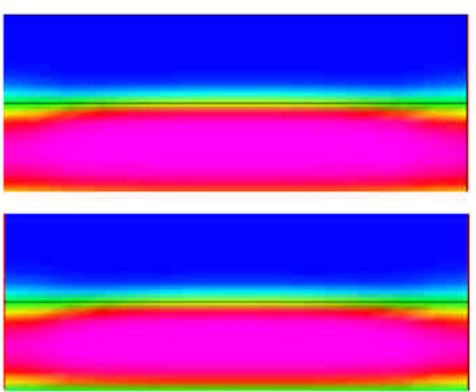
3.767E-005

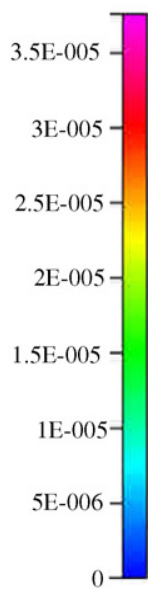

Fig. 9. The concentration contours of oxygen at the outlet of microchannel for both cases of volumetric flow rate $Q=1.0$ and $8.0 \mathrm{ml} / \mathrm{min}$ with three assigned values of $h_{\mathrm{c}}$ : (a) $Q=1.0 \mathrm{ml} / \mathrm{min}$ and $h_{\mathrm{c}}=100 \mathrm{~nm}$, (b) $Q=1.0 \mathrm{ml} / \mathrm{min}$ and $h_{\mathrm{c}}=500 \mathrm{~nm}$, (c) $Q=1.0 \mathrm{ml} / \mathrm{min}$ and $h_{\mathrm{c}}=900 \mathrm{~nm}$, (d) $Q=8.0 \mathrm{ml} / \mathrm{min}$ and $h_{\mathrm{c}}=100 \mathrm{~nm}$, (e) $Q=8.0 \mathrm{ml} / \mathrm{min}$ and $h_{\mathrm{c}}=500 \mathrm{~nm}$, and (f) $Q=8.0 \mathrm{ml} / \mathrm{min}$ and $h_{\mathrm{c}}=900 \mathrm{~nm}$. 


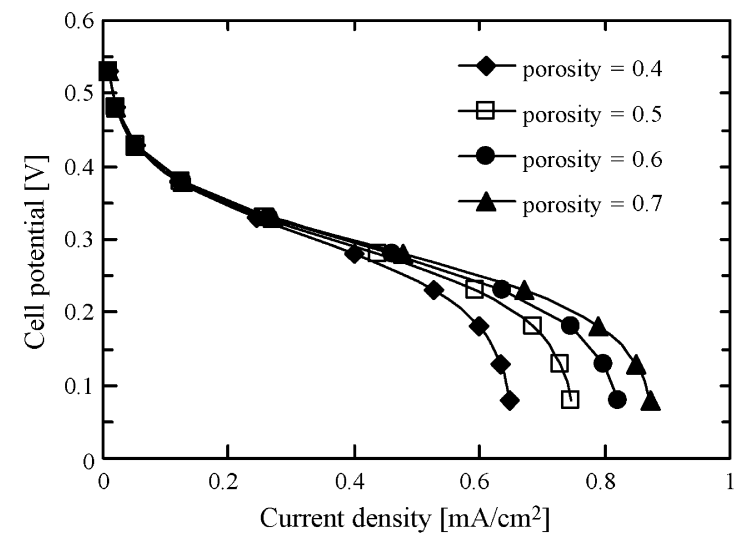

Fig. 11. The $I-V$ curves for four assigned porosities of cathode catalyst layer.

The porosity is also an important property of the catalyst layer. We can expect that the cell performance will be improved if the porosity of the catalyst layer increases, since the reactant is easier to penetrate into the catalyst layer to enhance the electrochemical reaction. Fig. 11 shows the variations of $I-V$ curves for four assigned values of porosity of the cathode catalyst layer. Obviously, the limiting current density increases gradually with the porosity. However, the increment between each case decreases from porosity 0.4 to 07 , which indicates that this effect diminishes gradually with the porosity. It is also noted that the porosity of the anode catalyst layer plays a minor role only on the cell performance.

\subsection{Effects of thicknesses of fuel and oxidant streams}

Theoretically, both fuel and oxidant streams in such a planar microchannel design should be as thin as possible to reduce the ohmic losses. However, the present results show that the mean velocity $\bar{U}$ of the liquid layer also plays an important role in the determination of cell performance. Fig. 12 demonstrates the variations of $I_{1}$ with $\bar{U}$ for two typical cases $d_{\mathrm{a}}=d_{\mathrm{c}}=95 \mu \mathrm{m}$ and $d_{\mathrm{a}}=d_{\mathrm{c}}=190 \mu \mathrm{m}$, where $d_{\mathrm{a}}$ and $d_{\mathrm{c}}$ are the thicknesses of anode and cathode liquid layers, respectively. It is found that when the mean velocity is less than $0.025 \mathrm{~m} / \mathrm{s}$, the losses caused by fuel

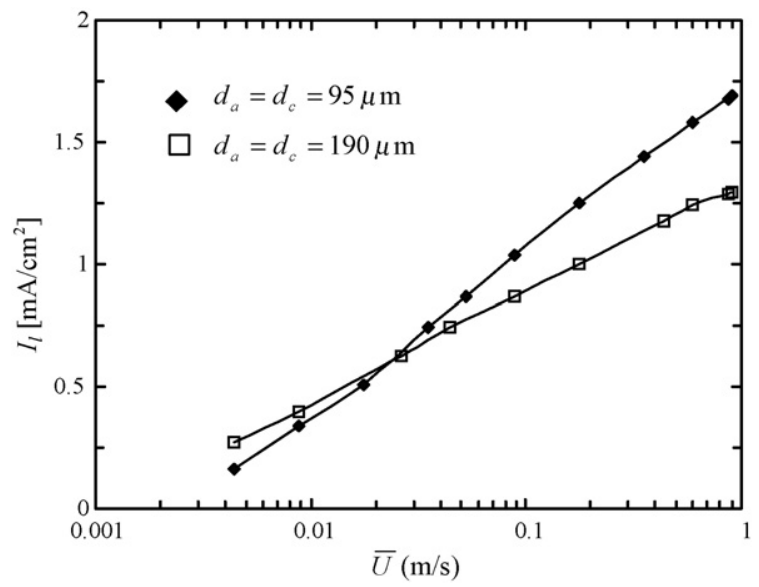

Fig. 12. The variations of limiting current density $I_{1}$ with mean velocity $\bar{U}$ for two cases $d_{\mathrm{a}}=d_{\mathrm{c}}=95 \mu \mathrm{m}$ and $d_{\mathrm{a}}=d_{\mathrm{c}}=190 \mu \mathrm{m}$.

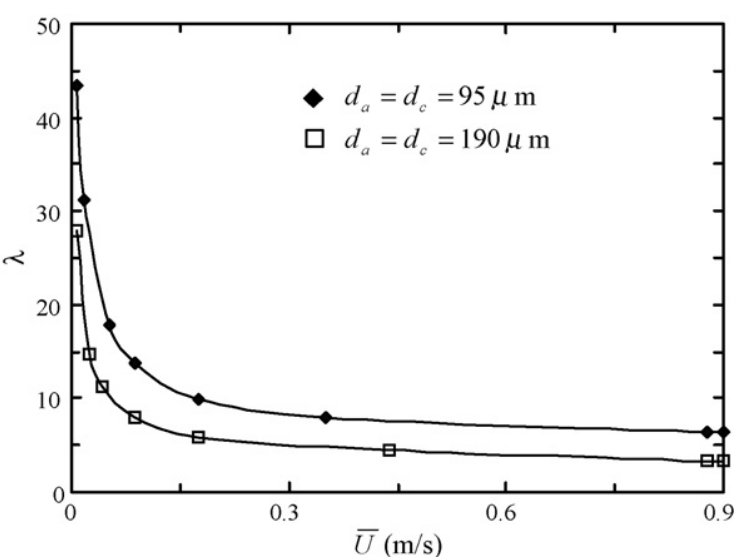

Fig. 13. The variations of oxidant consumption rate $\lambda$ with mean velocity $\bar{U}$ for two cases $d_{\mathrm{a}}=d_{\mathrm{c}}=95 \mu \mathrm{m}$ and $d_{\mathrm{a}}=d_{\mathrm{c}}=190 \mu \mathrm{m}$.

crossover are relatively more significant in a thinner stream for the same value of $\bar{U}$, which makes the lower cell performance. On the other hand, as $\bar{U}$ increases, the diffusive mixing between both streams diminishes gradually and thus the losses of fuel crossover decrease rapidly. Once $\bar{U}$ is large enough to avoid the occurrence of fuel crossover, the cell performance will be dominated primarily by the ohmic loss which depends on the stream thickness. Hence, as seen in Fig. 12, the case of smaller stream thickness will possess better performance as $\bar{U}$ is greater than $0.025 \mathrm{~m} / \mathrm{s}$. The corresponding oxygen consumption rates of both cases are illustrated in Fig. 13. Obviously, under the same mean velocity $\bar{U}$, the consumption rate $\lambda$ can be raised significantly by using a thinner microchannel. For example, at $\bar{U}=0.175 \mathrm{~m} / \mathrm{s}$, the value of $\lambda$ increases from 5.9 to $9.9 \%$ as the stream thickness decreases from 190 to $95 \mu \mathrm{m}$. This result also suggests that a thinner microchannel will benefit the transport of oxygen to the cathode as well as the improvement of cell performance.

Note that the ohmic losses are also related to the electric conductivity of the electrolyte. For dilute sulfuric acid solution, the electric conductivity is approximately proportional to its concentration [15]. However, since the cell performance is primarily limited by the transport of oxygen at the cathode side, the influence of the concentration of sulfuric acid solution is insignificant. For example, if we increase the concentration of sulfuric acid solution to $0.3 \mathrm{M}$, the corresponding $I-V$ curve will be quite close to that of the base case and the limiting current density will increase from 0.748 to $0.789 \mathrm{~mA} / \mathrm{cm}^{2}$ only.

\subsection{Effects of length of microchannel}

It can be predicted that the average current density will reduce if one uses a longer microchannel, since the depletion zone along the electrode surface grows gradually down the stream, which increases the transport resistance of reactant to the electrode. Fig. 14 shows the contour distribution of current density on the cathode electrode surface for three assigned channel lengths $L=3,5$, and $7 \mathrm{~cm}$. Clearly, the current density decreases gradually down the stream. Especially, in the regions near the upper and lower boundaries, the current density reduces more quickly 


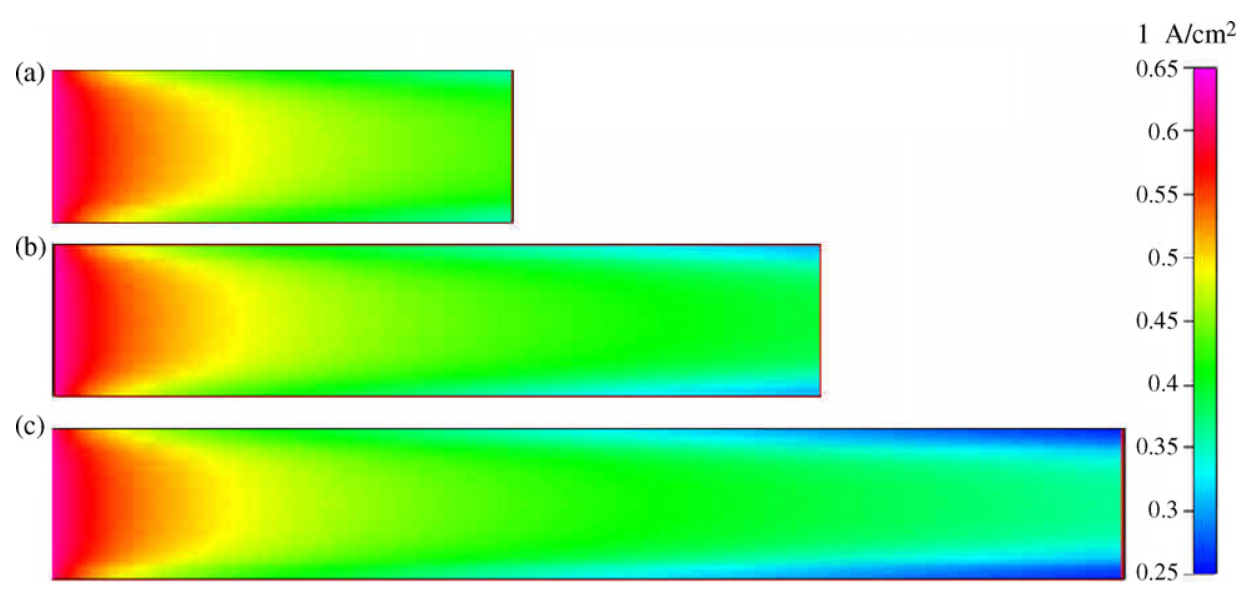

Fig. 14. The contours of current density on the cathode electrode surface for three assigned channel lengths at cell potential $0.3 \mathrm{~V}$, the vertical channel widths are magnified 10 times: (a) $L=3 \mathrm{~cm}$, (b) $L=5 \mathrm{~cm}$, and (c) $L=7 \mathrm{~cm}$.

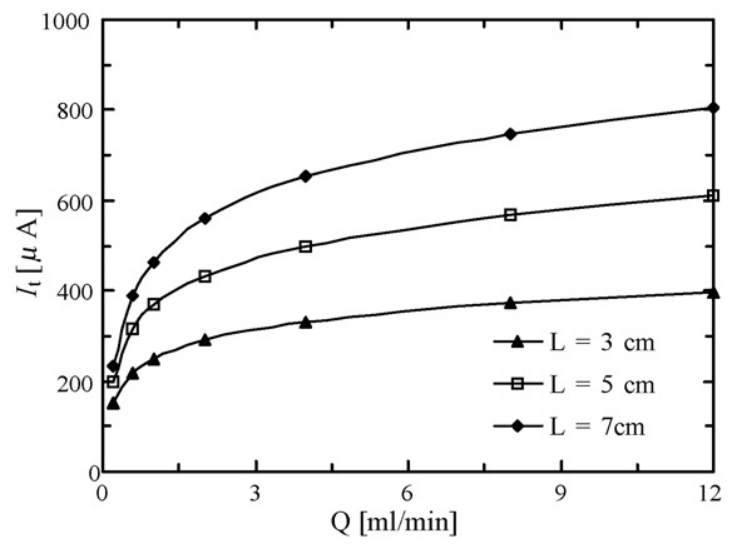

Fig. 15. The variations of total current output $I_{\mathrm{t}}$ with volumetric flow rate $Q$ for three assigned channel lengths at cell potential $0.15 \mathrm{~V}$.

due to the influences of lateral walls. Although the mean current density decreases with the channel length, the totally current output $I_{\mathrm{t}}$ of this microfuel cell still increases with the length as the results shown in Fig. 15. Besides, the oxygen consumption rate $\lambda$ also increases with the microchannel length. For example, the values of $\lambda$ at $Q=8.0 \mathrm{ml} / \mathrm{min}$ are 4.9 and $5.6 \%$ for both cases $L=5$ and $7 \mathrm{~cm}$, respectively. The current of increase is quite limited. Of course, we may use a longer microchannel to enhance the totally output of current and the utilization rate of oxygen. However, the diffusion zone between both anode and cathode streams also grows down the stream. In order to avoid the occurrence of fuel crossover, a higher flow rate is necessary while this may cause the possible leakage problem in practical apparatus. Moreover, the higher Reynolds number may exceed the critical value resulting in hydrodynamic instability and possibly severe fuel crossover, which has been observed in experimental study [2]. Therefore, for a given length of this planar microchannel, it should be critical to operate the microfuel cell with appropriate flow rate to obtain the optimal cell performance.

\section{Conclusions}

We have developed a theoretical model for a direct formic acid microfuel cell employing a design of planar microchan- nel. Both fuel and oxidant streams are separated by the nature of laminar flow within the microchannel under small Reynolds number and thus the membrane used in conventional PEMFC can be removed in such a membraneless design. The model has been solved numerically by a CFD package and the transport phenomena of reactants in the microchannel as well as their influences on cell performance have been examined in detail. The results confirm the experimental findings that the performance of this membraneless direct formic acid microfuel cell is limited by the low flux of oxygen transport to the cathode electrode. It can be improved by using a higher flow rate to reduce the transport resistance, or increasing the oxygen concentration of cathode stream as high as possible to enhance the amount of mass flux. A thicker cathode catalyst layer is also helpful to increase the cell performance. However, this effect fails once the thickness exceeds a certain critical value. The thickness of microchannel should be made as thin as possible to reduce the ohmic losses. But a higher flow rate is necessary to overcome the fuel crossover problem. Such an improvement also can significantly raise the consumption rate of oxygen.

The present results provide an overview for the characteristics of this planar membraneless microfuel cell and also benefit the further design work to optimize the cell performance. In comparison with the results of Y-shaped design [2], both cell performances are generally in the same order. Hence, it is important to find a more efficient way to raise the cell performance significantly. An interesting further study could be the other fuel systems such as direct methanol, $\mathrm{H}_{2} / \mathrm{O}_{2}$, and airbreathing membraneless microfuel cells including the effects of dual electrolytes. A comparison of performance between these membraneless microfuel cells will be helpful for the determination of which one will prevail in the practical applications in portable power sources.

\section{Acknowledgments}

The financial support for this research from National Science Council of Taiwan through the grants NSC 94-2212-E-132005, NSC 94-2212-E-002-008, and NSC 93-2212-E-002-029 are gratefully acknowledged. 


\section{References}

[1] R. Ferrigno, A.D. Stroock, T.D. Clark, M. Mayer, G.M. Whitesides, J. Am. Chem. Soc. 124 (2002) 12930.

[2] E.R. Choban, L.J. Markoski, A. Wieckowski, P.J.A. Kenis, J. Power Sources 128 (2004) 54.

[3] A. Bazylak, D. Sinton, N. Djilali, J. Power Sources 143 (2005) 57.

[4] M.H. Chang, F. Chen, N.S. Fang, J. Power Sources 159 (2006) 810.

[5] E.R. Choban, P. Waszczuk, P.J.A. Kenis, Electrochem. Solid-State Lett. 8 (2005) A348-A352.

[6] E.R. Choban, J.S. Spendelow, L. Gancs, A. Wieckowski, P.J.A. Kenis, Electrochim. Acta 50 (2005) 5390.

[7] R.S. Jayashree, L. Gancs, E.R. Choban, A. Primak, D. Natarajan, J. Am. Chem. Soc. 127 (2005) 16758.
[8] J.L. Cohen, D.A. Westly, A. Pechenik, H.D. Abruna, J. Power Sources 139 (2005) 96.

[9] J.L. Cohen, D.J. Volpe, D.A. Westly, A. Pechenik, H.D. Abruna, Langmuir 21 (2005) 3544.

[10] S. Motokawa, M. Mohamedi, T. Momma, S. Shoji, T. Osaka, Electrochem. Commun. 6 (2004) 562.

[11] J. Yeom, G.Z. Mozsgai, B.R. Flachsbart, E.R. Choban, A. Asthana, M.A. Shannon, P.J.A. Kenis, Sensors Actuators B 107 (2005) 882.

[12] S.M. Mitrovski, R.G. Nuzzo, Lab. Chip 6 (2006) 353.

[13] R.F. Ismagilov, A.D. Stroock, P.J.A. Kenis, G.M. Whitesides, H.A. Stone, Appl. Phys. Lett. 76 (2000) 2376.

[14] R.E. Meredith, C.W. Tobias, in: C.W. Tobias (Ed.), Advances in Electrochemistry and Electrochemical Engineering 2, Interscience Publishers, New York, 1962.

[15] D.R. Lide, CRC Handbook of Chemistry and Physics, CRC Press, 1975. 